\title{
Predição da gestão organizacional e do perfil inovador das empresas de pequeno porte: análise à luz de Cadeias de Markov
}

\author{
Prediction of organizational management and the \\ innovative profile of small businesses: Markov \\ Chain light analysis..
}

\author{
André Marques Cavalcanti²
}

Auristela Maria da Silva ${ }^{1}$

${ }^{1}$ Universidade Federal de Pernambuco Endereço: Av. Prof. Moraes Rego, 1235 - Cidade Universitária, Recife - PE - Brasil - CEP: 50670-901

Mestre em Ciências Administrativas pela Universidade Federal Rural de Pernambuco (2018). Atualmente é engenheira eletrônica da Universidade Federal de Pernambuco, atuando Núcleo de Inovação, Empreendedorismo e Sustentabilidade (NIES) do Centro de Ciências Sociais Aplicadas/UFPE. auristela.msilva@gmail.com

${ }^{2}$ Universidade Federal de Pernambuco Endereço: Av. Prof. Moraes Rego, 1235 - Cidade Universitária, Recife - PE - Brasil - CEP: 50670-901 Doutorado em Engenharia Elétrica pela Universidade Federal de Pernambuco (2004). Atualmente é professor adjunto da

UFPE e coordenador do Núcleo de Inovação, Empreendedorismo e Sustentabilidade (NIES) do Centro de Ciências Sociais Aplicadas/UFPE. andremarques2008@gmail.com

\begin{abstract}
Resumo
Neste artigo apresentar-se-á um modelo probabilístico que contribui para o estudo da dinâmica do comportamento das Micro e Pequenas Empresas com relação ao seu perfil inovador e organizacional em longo prazo. O modelo utilizado corresponde a uma Cadeia de Markov em tempo discreto, que ao definir os níveis de maturidade (estados) das empresas e a obtenção das probabilidades de transição em um passo correspondentes entre níveis, permite descrever e prever os estados futuros dessas organizações. Os diferentes estados utilizados são baseados no grau de desenvolvimento organizacional (GO) e o grau de inovação (GI) de 60 empresas, obtidos a partir do Projeto Agentes Locais de Inovação do Serviço Brasileiro de Apoio às Micro e Pequenas Empresas [SEBRAE] (2015). A amostra é igualmente distribuída em três grupos de 20 empresas dos setores da indústria de alimentos, móveis e confecções do estado de Pernambuco. Mostrou-se que em longo prazo, dado que não haja nenhuma ação do gestor da empresa para melhorar o estado da mesma e/ou um evento externo, as empresas, em sua maioria, apresentam um perfil de inovadoras ocasionais.
\end{abstract}

Palavras chave: Inovação. Gestão. Perfil inovador. Micro e Pequenas Empresas. Cadeias de Markov.

\begin{abstract}
This paper will present a probabilistic model that contributes to the study of the dynamics of Micro and Small Businesses behavior in relation to its innovative and organizational profile in the long term. The model used corresponds to a Markov Chain in discrete time, which in defining the levels of maturity (states) of the companies and obtaining the probabilities of transition in a corresponding step between levels, allows to describe and predict the future states of these organizations. The different states used are based on the degree of organizational development (GO) and the degree of innovation (GI) of 60 companies, obtained from the Local Agents of Innovation Project of the Brazilian Service of Support to Micro and Small Companies [SEBRAE] (2015). The sample is also distributed in three groups of 20 companies in the food, furniture and clothing industries in the state of Pernambuco. It has been shown that in the long run, since there is no action by the company manager to improve the state of the company and / or an external event, most companies present an occasional innovative profile.
\end{abstract}

Keywords: Inovation. Management. Profile innovator. Small enterprises. Markov Chain. 


\section{Introdução}

As Micro e Pequenas Empresas (MPE) vêm progressivamente aumentando sua relevância na economia brasileira. Constatou-se que esta atuação era de 21\% em 1985, aumentou para 23\% em 2001 e para $27 \%$ em 2011. Esta participação cresceu tanto em serviços como no comércio, tendo se reduzido um pouco na atividade industrial, onde predominam médias e grandes empresas que se beneficiam de economias de escala (SEBRAE, 2014).

Entretanto, para afastarem as ameaças impostas pelo mercado e se manterem competitivas, as MPE precisam inovar. Cada organização deve ser capaz de reconhecer os seus pontos fortes e fracos para estabelecer estratégias inovadoras, seja através da implementação de uma nova ou significativamente melhoria na empresa, de um novo produto, processo, método organizacional ou de marketing com o objetivo de reforçar a sua posição competitiva, aumentar a performance, ou o conhecimento. Assim, a inovação passa a ser um elemento fundamental para as organizações que buscam a continuidade no mercado e obtenção de diferencial competitivo

Leone e Leone (2012) explicam que uma das dificuldades em estudar as micro, pequenas e médias empresas se baseia na heterogeneidade que existe entre elas. Este pode ser um dos fatores que explicam a dificuldade dessas empresas de promover inovações necessárias para a sobrevivência e crescimento sustentável. Outro ponto que deve ser observado é que classificar o que é uma micro e pequena empresa é um assunto complexo (Oliveira, Terence, \& Escrivão Filho, 2010; Leone \& Leone, 2012).

Motivados pelas discussões relativas à capacidade de inovar das MPE, buscou-se utilizar um modelo de predição de estado futuros de empresas de pequeno porte para o grau de desenvolvimento organizacional (GO) e o grau de inovação (GI), a partir do Radar de Inovação definido por Sawhney, Wolcott e Arroniz (2006) e ampliado por Bachmann (2008). O modelo utilizado tem como base a teoria de Cadeia de Markov em tempo discreto, comumente usado no campo da pesquisa operacional para descrever e prever o comportamento de certos sistemas em condições de incerteza ao longo do tempo. A utilização deste modelo já é amplamente utilizado para modelagem dinâmica em diversas áreas, tais como: sistemas de espera, controle de estoques, manutenção e substituição de equipamentos e em apoio à tomada de decisões na administração, engenharia e medicina.

Os resultados obtidos, podem ser utilizados por gestores das organizações e por gestores de políticas públicas para criar vantagem competitiva nas MPE, através de ações que tornem as empresas mais competitivas.

\section{Referencial Teórico}

Neste capítulo são apresentadas definições de inovação, vantagem competitiva através da inovação e como mesurar a inovação. São citadas algumas formas de mensuração da mesma, começando pelo critério proposto por Schumpeter (1984), chegando às modificações sugeridas por Bachmann (2008) com base nos estudos de Sawhney et al. (2006). Na vantagem competitiva enfatizamos a estratégia da inovação para desenvolver e tornar as empresas mais robustas frente aos eventos externos. Descreveremos, também, a ferramenta de cadeias de Markov que será utilizada para descrição e predição de estados futuros das empresas. 


\subsection{Inovação}

Simantob e Lippi (2003) afirmam que a inovação é uma iniciativa, modesta ou revolucionária, que surge como uma novidade para a organização e para o mercado e que, aplicada na prática, traz resultados econômicos para a empresa - sejam eles ligados à tecnologia, gestão, processos ou modelo de negócio. Acrescentam dizendo que o cerne da inovação está no consumidor - é preciso atender às suas necessidades para efetivamente criar valor.

Os dados apresentados no relatório "Desenvolvimento Tecnológico e Inovação nas Microempresas e Pequenas Empresas: Fatores de Influência", elaborado pelo Fórum Permanente das Microempresas e Empresas de Pequeno Porte (2007), indicam fatores que dificultam o desenvolvimento tecnológico e a inovação nas MPE. A pesquisa destaca alguns fatores que contribuem para este cenário: baixo nível de investimento em P\&D adequado à realidade das MPE, pouca tradição em investir em desenvolvimento tecnológico, reduzido conhecimento técnico da parte das MPE, dependência de fornecedores dos principais insumos tecnológicos inovadores, alto custo para a aquisição das inovações, dificuldade para acessar linhas de crédito para adquirir equipamentos; a pouca aproximação dos centros de tecnologias; carência de infraestrutura física e de pessoal adequados, entre outras.

Para Andreassi (2003), as pequenas empresas, com exceção das empresas de base tecnológica, têm a sua atividade de inovação ligada à linha de produção, em atividades relacionadas com a adaptação de tecnologias adquiridas do exterior da firma ou em pequenas melhorias implementadas pela área de engenharia industrial. Andreassi e Sbragia (2002), em sua pesquisa sobre os fatores que geram inovação nas pequenas, médias e grandes empresas, concluíram que o número de funcionários alocados à área de P\&D é o grande diferencial para as empresas consideradas inovadoras. E neste cenário, as grandes empresas levam vantagem, pois possuem uma infraestrutura propícia e recursos para investir em P\&D.

Entretanto, Bachmann e Destefani (2008) argumentam que os indicadores tradicionalmente utilizados para medir inovação nas organizações, tais como investimento em P\&D e número de patentes, não são adequados para serem utilizados com as MPE. Segundo os autores, os principais motivos para a falta de conformidade desses indicadores na realidade das MPE são: os gastos em P\&D, como regra, não são apropriados adequadamente; muitas inovações não são fruto direto de P\&D, mas trazidas por fornecedores, copiadas de concorrentes ou resultantes de insights eventuais dos empreendedores ou seus colaboradores; e por fim, dado ao custo e ao baixo reconhecimento de sua importância, raramente, as inovações são objeto de patenteamento.

Campos e Campos (2013) apontam no seu estudo de casos múltiplos realizado com pequenas empresas que a inovação não é privilégio das grandes organizações e que elas podem fomentar inovações incrementais com poder de ampliar seus mercados. Destacam, no entanto, que a falta de processo definidos na gestão desses resultados faz com que esses indicadores não sejam potencializados. Estes autores corroboram com o modelo proposto por Schumpeter (1984), abordando dimensões para a inovação. Segundo Schumpeter (1984), a inovação pode surgir sob a dimensão de um novo produto, novo processo, pela procura de novos mercados, desenvolvimento de novas fontes de matérias primas ou novas estruturas de mercado. 
Neste contexto, esta pesquisa assume, tal como Reichert, Camboim e Zawislak (2015), que a inovação é uma novidade, cuja aplicação proporciona resultados organizacionais positivos, que elevam as organizações a posições superiores em relação aos seus pares, levando as empresas a obter vantagem competitiva.

\subsection{Vantagem competitiva através da inovação}

Segundo Martín e López (2007, p.267), entende-se por vantagem competitiva como qualquer característica da empresa que a diferencia das demais, colocando-a numa posição relativa de superioridade para competir.

Na concepção de Akis (2015), a qualidade e os custos de produção eram determinantes do poder de competitividade, porém nos dias atuais, estes fatores foram substituídos gradativamente pela inovação.

Tidd et al. (2008, p.30) associam a vantagem competitiva a capacidade da empresa inovar. Segundo esses autores, sejam quais forem as condições tecnológicas, sociais ou mercadológicas envolvidas, a chave para se criar e manter vantagem competitiva tende a pertencer àquelas organizações que inovam continuamente. Ainda, segundo esses autores, as organizações de grande porte estão aptas a desenvolverem inovações em larga escala, enquanto as MPE geralmente adotam estratégias de inovação por meio da aquisição de tecnologia. Assim, empresas de diferentes portes buscam formas distintas para inovar.

Para Vargas, Estrada e Gómez (2016, p.49) “a inovação é um fator determinante para o desempenho da empresa, que por conseguinte, é essencial para as empresas repensarem as suas estratégias para dar maior importância às ações de inovação, que permitirá alcançar vantagens competitivas e desempenho superior". Nesse sentido, as empresas podem adotar estratégias de inovação que as coloquem em um patamar diferenciado frente aos seus concorrentes.

Titu et al. (2015) defendem que o sucesso das organizações depende da sua capacidade de se adaptar às rápidas alterações do ambiente. Assim, a inovação deve fazer parte da cultura da empresa, de forma que seja empreendida no longo prazo por meio do desenvolvimento de um espírito inovador

Na perspectiva de Alsaaty (2011), a estratégia da inovação para as pequenas empresas - em função da limitação de recursos e da sua necessidade de atingir o crescimento - é o mercado, ou seja, os clientes são o foco da empresa para as suas atividades inovadoras. Corroborando com esta abordagem, Zehir et al. (2015) e Vargas et al. (2016) concluem em suas pesquisas que as MPE conseguem obter vantagens competitivas através da melhoria da capacidade de inovação orientada para o mercado. Na mesma direção desses autores, Ceretta et al. (2016) defendem que a ampliação mercadológica gera vantagens competitivas à organização.

Nesse sentido, o projeto ALI (SEBRAE, 2015) traz uma metodologia que tem o potencial de contribuir para o alcance de vantagem competitiva, uma vez que é realizado um diagnóstico para apontar os pontos fortes e fracos das MPE, propondo ações de melhoria para as dimensões que a empresa precisa aprimorar ou desenvolver.

\subsection{Como mensurar a inovação}

Conforme destacado em Gamal, Salah e Elrayyes (2011), há duas grandes vertentes para a mensuração da inovação. A primeira é baseada nos investimentos em projetos de $P \& D$, os quais têm como resultados 
esperados patentes e inovação tecnológica. Esta abordagem delimita o número de empresas que podem gerar inovação, uma vez que as pequenas e médias não possuem um departamento de P\&D, nem recursos humanos qualificados para atuar na área. A segunda vertente traz uma abordagem macro da inovação através de indicadores econômicos. É o caso do Manual de Oslo (2005), da Pesquisa de Inovação [PINTEC] (2005) do Instituto Brasileiro de Geografia e Estatística (IBGE), do Placar de Inovação Europeu [EIS] (2007) e do Radar da Inovação de Sawhney et al. (2006).

Schumpeter (1984) propõe uma ferramenta que relaciona as dimensões pelas quais uma empresa pode procurar caminhos para inovar. O modelo reúne quatro dimensões principais: ofertas criadas, clientes atendidos, processos e praça. Segundo o autor, tais dimensões compõem a base de referência para se determinar a inovação.

Sawhney et al. (2006) perceberam que as empresas procuram outros caminhos para alcançar níveis mais elevados de competitividade por meio da inovação. Esses autores propõem uma ferramenta denominada Radar de Inovação, a qual relaciona 12 dimensões pelas quais uma empresa se desenvolve em uma proposta de inovar seus produtos, processos e/ou gestão. Os autores consideram quatro dimensões principais: as ofertas criadas pela organização, os clientes atendidos, os processos usados e os locais utilizados para dispor suas ofertas ao mercado. No entanto, eles constataram que as empresas procuram outros caminhos para alcançar patamares mais elevados de competitividade por meio da capacidade de inovar, assim incorporaram mais oito dimensões: plataforma, marca, soluções, relacionamento, agregação de valor, organização, cadeia de fornecimento e rede. Considerando a influência do clima organizacional ao desenvolvimento de uma cultura inovadora, Bachmann (2008) aprimorou a ferramenta, acrescentando a dimensão "ambiência inovadora".

O modelo proposto por Sawhney et al.(2006) e ampliado por Bachman (2008) possibilita uma mensuração mais abrangente da inovação organizacional. Para analisar o nível de maturidade das empresas com relação à inovação, utilizou-se a escala de Bachmann (2008). Este autor propõe uma medição objetiva do grau de inovação através de uma escala para 3 situações, variando de 1 a 5, visando classificar as empresas em "Pouco ou nada inovadoras" - nível 1, "Inovadoras ocasionais" - nível 3 e "Inovadoras sistêmicas" - nível 5. Esta escala também atende a recomendação do Manual de Oslo (2005), que estabelece que os pontos de vista das empresas sejam registrados, ou em bases binárias (importante/não importante), ou com uma estreita faixa de respostas possíveis (irrelevante até muito relevante).

Uma vez que o Radar de Inovação apresenta essa visão mais ampliada de inovação, o Programa Agentes Locais de Inovação (SEBRAE, 2015) passou a adotá-lo na avaliação do grau de inovação das empresas.

\subsection{Cadeias de Markov}

Nesta seção introduzir-se-á os principais conceitos relativos à Cadeia de Markov e utilizar-se-á como referência as obras de Ross (2003), Sullivan (2006) e Taha (2008).

Nos mais diversos ramos da ciência, as transições de estados baseadas em probabilidades são utilizadas para analisar fenômenos em diversas áreas do conhecimento. No caso em que a probabilidade de transição depende apenas do estado em que o fenômeno se encontra e do estado seguinte, o processo é chamado processo de Markov e uma sequência de estados seguindo este processo é denominado Cadeias de Markov. 
Os modelos para processos que se desenvolvem ao longo do tempo, de uma forma probabilística, são os chamados processos estocásticos. Um processo estocástico é definido como uma coleção de variáveis randômicas $(X(t))$, indexadas por um parâmetro t pertencente a um conjunto T. Desta forma $X(t)$ representa uma variável mensurável variando no tempo.

As Cadeias de Markov são um tipo específico de processo estocástico, possuindo uma propriedade markoviana na qual a predição de eventos futuros depende apenas do estado atual do processo. Esta propriedade é definida pela equação a seguir:

$$
\mathbb{P}\left[X_{n}=x_{n} \mid X_{0}=x_{0}, \cdots, X_{n-1}=x_{n-1}\right]=\mathbb{P}\left[X_{n}=x_{n} \mid X_{n-1}=x_{n-1}\right]
$$

Atualmente, várias áreas de conhecimento utilizam as Cadeias de Markov para descrever e prever seus estados futuros, entre elas estão: modelo de processo de decisão (Nuñez, 2004), modelo econométrico (Silos, 2006), modelo de controle de praga na lavoura (Marcos, 2014), modelo epidêmico (Estrada, 2015; Fidalga, 2016), análise da confiabilidade na área de perfuração de poços de petróleo (Oliveira, 2016), entre outras. Neste contexto, esta pesquisa usará esta ferramenta para descrição e predição dos estados futuros nas áreas organizacionais e de inovação de empresas de pequeno porte.

\section{Metodologia}

Esta pesquisa é exploratória e quantitativa, sendo desenvolvida a partir dos diagnósticos de inovação e organizacional, baseada no Radar de Inovação do projeto ALI (SEBRAE, 2015). A amostra é formada por 60 empresas dos setores da indústria de alimentos, móveis e confecções do estado de Pernambuco, sendo obtida de modo não probabilístico por conveniência de adesão das empresas ao projeto Agentes Locais de Inovação (SEBRAE, 2015). A amostra é igualmente distribuída em três grupos de 20 empresas, considerando-se a diferença natural entre os setores, sobretudo, no tocante às possibilidades de inovar.

Os agentes de inovação do projeto Sebrae (2015) utilizaram dois tipos de formulários: um para diagnóstico da inovação, composto por 40 construtos agrupados em 13 dimensões, das quais 12 coincidem com as dimensões da inovação propostas por Sawhney et al. (2006) e mais a dimensão ambiência inovadora proposta por Bachmann (2008), sintetizadas no quadro 1; e um para diagnóstico empresarial baseado no Modelo de Sistema de Gestão (MEG) da Fundação Nacional da Qualidade [FNQ] (2016), composto por oitos dimensões sintetizadas no quadro 2. De acordo com o MEG, a organização pode ser considerada como um sistema orgânico, adaptável ao ambiente externo. Os vários elementos do modelo encontram-se imersos num ambiente de informação e conhecimento, e relacionam-se de forma harmônica e integrada, voltados para a geração de resultados. 
Quadro 1 - Dimensões do radar de inovação e suas definições

\begin{tabular}{|c|c|}
\hline Dimensão & Definição \\
\hline Oferta & Desenvolvimento de produtos e serviços com característico inovador. \\
\hline Plataforma & $\begin{array}{l}\text { Refere-se a capacidade do sistema produtivo de adaptar-se a novas } \\
\text { demandas e produtos. }\end{array}$ \\
\hline Marca & $\begin{array}{l}\text { Relaciona-se as maneiras de como as MPEs transmitem seus valores aos } \\
\text { clientes. }\end{array}$ \\
\hline Clientes & Identificar novas demandas, nichos e necessidades. \\
\hline Soluções & Capacidade de sistematizar meios de solucionar as dificuldades dos clientes. \\
\hline Relacionamento & Relaciona-se a experiência dos seus clientes. \\
\hline Agregação de valor & $\begin{array}{l}\text { Otimizar a capacitação dos valores referente aos seus produtos pelo público } \\
\text { externo. }\end{array}$ \\
\hline Processos & $\begin{array}{l}\text { Revisão dos procedimentos produtivos a fim de permitir a eficiência } \\
\text { operacional. }\end{array}$ \\
\hline Organização & Melhorar a estrutura organizacional. \\
\hline Cadeia de fornecimento & $\begin{array}{l}\text { Otimizar a infraestrutura de logística com ambiente interno e externo } \\
\text { empresarial. }\end{array}$ \\
\hline Presença & Refere-se a difusão dos produtos em vários pontos e em novos mercados. \\
\hline Rede & Buscar a eficiência na comunicação organizacional. \\
\hline Ambiência inovadora & $\begin{array}{l}\text { Relaciona-se com o capital humano da empresa e sua relação com a cultura } \\
\text { inovadora. }\end{array}$ \\
\hline
\end{tabular}

Fonte: Adaptado de Sawhney, Wolcott e Arroniz (2006) e Bachmann (2008).

Quadro 2 - Dimensões do radar de organização e suas dimensões

\begin{tabular}{|c|c|}
\hline Dimensão & Definição \\
\hline Liderança & $\begin{array}{l}\text { Atuação dos líderes de forma aberta e ética, com disponibilização de } \\
\text { informação e inspirando a cultura empreendedora. }\end{array}$ \\
\hline Estratégias e Planos & $\begin{array}{l}\text { Refere-se ao desenvolvimento de estratégias e planos e a sua } \\
\text { implementação e avaliação. }\end{array}$ \\
\hline Clientes & Entender as expectativas de clientes e suas demandas. \\
\hline Sociedade & $\begin{array}{l}\text { Relaciona-se com as novas demandas pela sociedade, exemplo } \\
\text { sustentabilidade. }\end{array}$ \\
\hline Informações e Conhecimentos & $\begin{array}{l}\text { Relaciona-se a uma estrutura para o acesso de informação e } \\
\text { compartilhamento de ideias. }\end{array}$ \\
\hline Pessoas & Refere-se ao capital intelectual na empresa. \\
\hline Processos & $\begin{array}{l}\text { Relaciona-se a organização com a formulação e padronização de } \\
\text { processos. }\end{array}$ \\
\hline Resultados & Busca-se analisar e compreender os resultados alcançados. \\
\hline
\end{tabular}

Com os dados dos diagnósticos empresarial e inovação, obteve-se o grau de desenvolvimento organizacional $(G O)$ e o grau de inovação $(G I)$ das empresas participantes da pesquisa. Os valores de $G O$ e $G I$ de cada empresa são calculados a partir da divisão dos valores das médias obtidas em cada dimensão pelo número total de dimensões. Neste cenário, há 8 (oito) dimensões para GO e 13 (treze) dimensões pata GI. Desta forma, o grau de organizacional e o grau de inovação médios são obtidos a partir da divisão do somatório dos valores das médias de cada dimensão de todas as empresas que fazem parte da amostra pelo número total de dimensões.

Para o objetivo principal da pesquisa, que é a descrição e predição de estados futuros de gestão e inovação das empresas, partindo-se de um estado atual, utilizou-se a ferramenta de Cadeias de Markov. Este é um tipo especial de processo estocástico onde as distribuições de probabilidade para os passos futuros do 
processo dependem somente do estado presente, desconsiderando como o processo chegou a tal estado (Ross, 2003, pp. 181-185).

Os estados da Cadeia de Markov foram definidos observando o conjunto de dados e o que se pretende analisar, no caso desta pesquisa analisar-se-á os estados dos graus de inovação e gestão das empresas. Para análise desta pesquisa, dividiu-se os dados em 04 (quatro) estados de GO e em 04 (quatro) estados de GI, considerando o estado 1 (um) o mais elementar e o estado 4 (quatro) o mais elevado.

A quantificação das transições para construção das matrizes de um passo de GO, GI e do par GOxGl para Cadeia de Markov foi feita através da ocorrência das frequências relativas, estratégia que é mais adequada ao perfil dos dados utilizados. Assim, a probabilidade de transição pij resulta do cálculo do quociente entre o valor total das transições do estado i para o estado j e do total dessas transições que simplesmente começam em i, considerando todas essas transições que ocorrem nos ciclos do conjunto de dados de GO e Gl.

Após a montagem das matrizes de transição de um passo, utilizou-se a propriedade da Cadeia de Markov regular para calcular-se, algebricamente, a probabilidade de após n passos, uma empresa estar em um determinado estado. Para o cálculo da probabilidade de transição em n passos, utilizou-se as Equações de Chapman-Kolmogorov (Ross, 2003, pp. 359-363). Com estes resultados, analisou-se os dados dos estados futuros das empresas para gestão e inovação.

\section{Resultados}

$\mathrm{Na}$ análise dos dados das empresas pesquisadas, buscou-se realizar a descrição e predição de estados futuros dos graus de desenvolvimento organizacional (GO) e de inovação (GI) dos três grupos de 20 empresas dos setores da indústria de alimentos, móveis e confecções do estado de Pernambuco.

Obtiveram-se os dados em três momentos temporais distintos, denominados Ciclo 0, Ciclo 1 e Ciclo 2 do Projeto ALI (SEBRAE, 2015). Em cada ciclo são realizados dois diagnósticos para cada empresa, gerando valores de GO e Gl. Com essa análise são sugeridas melhorias nas dimensões que a empresa precisa avançar para se tornar mais competitiva. Após 03 (três) meses o agente de inovação volta a realizar o diagnóstico, e dependendo se as ações foram implantadas ou não, a empresa pode ter permanecido no mesmo estado, retroagido ou avançado nas áreas organizacional e de inovação.

A partir dos dados pesquisados, calculamos a matriz de transição ou matriz estocástica de Gl em um passo (2), obtendo-se as probabilidades a seguir:

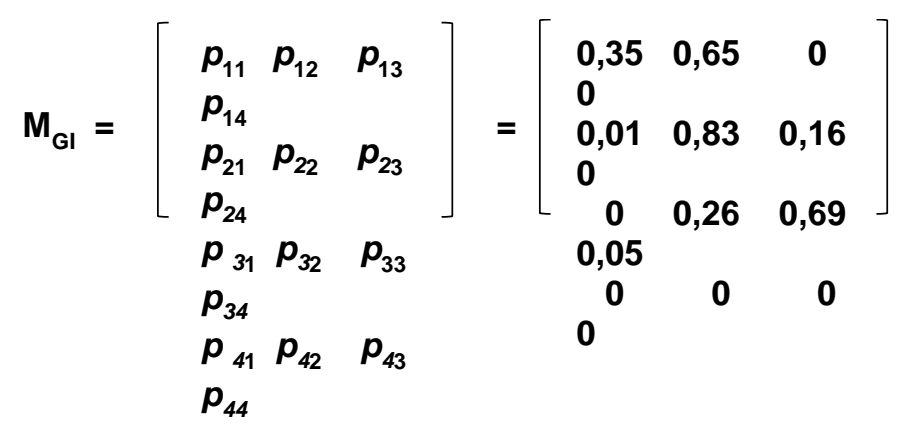

Com os dados desta matriz, analisou-se que uma empresa no estado GI1 tem a probabilidade de 35\% de permanecer neste estado; 65\% de passar para o estado GI2. Da mesma forma, uma empresa que esteja no estado 
GI2 tem 1\% de probabilidade de cair para o estado GI1; 83\% de permanecer no estado atual e 16\% de migrar para o estado GI3. Verifica-se que as empresas nos estados de GI3 têm as seguintes probabilidades: 26\% de retroceder para GI2; 69\% de se manter em GI3; 5\% de avançar para GI4. Esta análise baseia-se na premissa que não haverá nenhuma ação do gestor e/ou evento externo que modifique o estado da empresa. As probabilidades das matrizes podem ser usadas no planejamento da empresa para adquirir vantagem competitiva. Sendo assim, se uma empresa está no estado GI3 e quer avançar para GI4, a mesma precisa implementar ações para atingir este objetivo.

Note-se que, inicialmente, são quatro as possibilidades para o estado inicial das empresas: $\mathrm{Gl}=1, \mathrm{Gl}=2$, $\mathrm{Gl}=3$ e $\mathrm{Gl}=4$. Pode-se descrever este cenário, respectivamente, por meio de quatro matrizes linha, conforme descrição a seguir (3):

GI1 GI2 GI3 GI4

GI1 GI2 GI3 GI4

Gl1 GI2 GI3 GI4 GI1 GI2 GI3 GI4

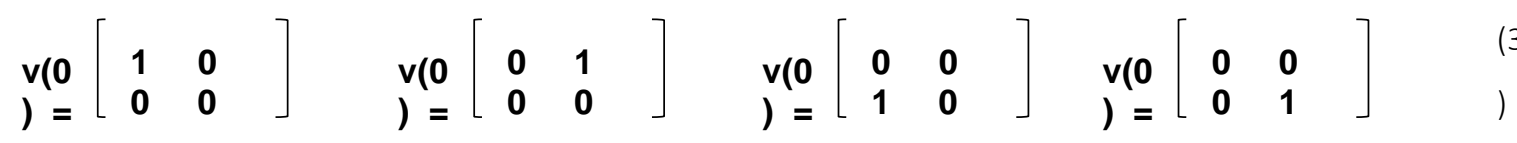

Para calcular as probabilidades de uma empresa estar em $\mathrm{Gl}_{3}$ em 3 passos ( $n=3$ ), dado que hoje a mesma está em Gl2, multiplicando-se o vetor linha de Gl2 pela matriz de transição de GI (Ross, 2003, p. 359-363) (4):

$$
\begin{aligned}
& v(3)=\left[\begin{array}{llll}
0 & 1 & 0 & 0
\end{array}\right] \cdot\left[\begin{array}{cccc}
0,35 & 0,65 & 0 & 0 \\
0,01 & 0,83 & 0,16 & 0 \\
0 & 0,26 & 0,69 & 0,05 \\
0 & 0 & 0 & 0
\end{array}\right] 3 \\
& v(3)=\left[\begin{array}{llll}
0,01 & 0,69 & 0,28 & 0,01
\end{array}\right]
\end{aligned}
$$

Portanto, daqui a três passos, haverá aproximadamente, 1\% de probabilidade de a empresa regredir para GI1, 69\% de permanecer em GI2, 26\% e 1\% de avançar para GI3 e GI4, respectivamente. Estes resultados confirmam que a maioria das empresas da amostra estão representadas por GI 2 e 3, demonstrando um perfil inovador ocasional.

Com relação aos dados do Grau de Organização, gerou-se a matriz de transição MGO de um passo (5), e verificou-se que há $77 \%$ de probabilidade da empresa que esteja em GO1 permanecer neste estado; 22\% de migrar para GO2; 1\% de passar para GO3 e 0\% de atingir GO4. Para as empresas que estão atualmente no estado GO2, há 2\% de probabilidade de ir para o GO1; 85\% de permanecer no estado GO2; 13\% de migrar para o estado GO3. No que se refere a GO3, as empresas apresentam 50\% de probabilidade de ir para GO2 e 50\% de permanecer em GO3.

$$
\mathrm{M}_{\mathrm{GO}}=\left[\begin{array}{llll}
p_{11} & p_{12} & p_{13} & p_{14} \\
p_{21} & p_{22} & p_{23} & p_{24} \\
p_{31} & p_{32} & p_{33} & p_{34} \\
p_{41} & p_{42} & p_{43} & p_{44}
\end{array}\right]=\left[\begin{array}{cccc}
0,77 & 0,22 & 0,01 & 0 \\
0,02 & 0,85 & 0,13 & 0 \\
0 & 0,50 & 0,50 & 0 \\
0 & 0 & 0 & 0
\end{array}\right]
$$


Da mesma forma que se analisou GI, calculou-se as probabilidades de uma empresa estar em GO3 em quatro passos (6), dado que hoje a mesma está no estado GO2, conforme cálculos a seguir (Ross, 2003, p. 359363):

$$
\begin{aligned}
& \mathbf{v}(4)=\left[\begin{array}{llll}
0 & 1 & 0 & 0
\end{array}\right] \cdot\left[\begin{array}{cccc}
0,77 & 0,22 & 0,01 & 0 \\
0,02 & 0,85 & 0,13 & 0 \\
0 & 0,50 & 0,50 & 0 \\
0 & 0 & 0 & 0
\end{array}\right]^{4} \\
& v(4)=\left[\begin{array}{llll}
0,04 & 0,76 & 0,20 & 0
\end{array}\right]
\end{aligned}
$$

Verificou-se que daqui a 4 passos futuros haverá, aproximadamente, $4 \%$ de probabilidade da empresa se posicionar em GO1; 76\% em GO2; 28\% em GO3 e 0\% da empresa migrar para G04.

Essas probabilidades de GI e GO para períodos posteriores podem ser usadas pelos gestores como um cenário futuro, dada as condições das empresas retratadas nos três ciclos do Projeto ALI. Com esses dados as empresas podem se planejar para alcançar estados mais avançados de inovação e gestão, ganhando vantagem competitiva.

Para a predição de estados em longo prazo das empresas, utilizaram-se as matrizes de transições de um passo para GO e GI. Para realizar a predição em longo prazo, a matriz de transição deve satisfazer as propriedades de uma matriz de Markov regular, que é uma matriz que não possui entradas nulas.

Para o cálculo de Gl em longo prazo, utilizou-se a matriz de transição de um passo de GI (2). Observa-se que a linha GI4 possui todas as entradas nulas, assim é preciso verificar se esta é uma matriz de Markov regular para depois prever os estados em longo prazo. Observa-se, ainda, que apenas duas empresas que estavam no estado 3, atingiram o nível 4 (p34 =0,05). Como essas empresas representam 3\% do total da amostra, vamos desprezá-las nesta análise.

Desta forma, pode-se eliminar a última linha e coluna, correspondente ao nível 4, e ajustando os valores da linha 3, obter a seguinte matriz de transição de um passo reduzida:

$$
N M_{G I}=\left[\begin{array}{ccc}
0,35 & 0,65 & 0 \\
0,01 & 0,83 & 0,16 \\
0 & 0,28 & 0,72
\end{array}\right]
$$

Neste caso NMGI (7) possui uma entrada nula, entretanto fazendo-se o produto (NMGI)2, verificou-se o seguinte resultado:

$$
\mathrm{NM}_{\mathrm{Gl}}^{2}=\left[\begin{array}{ccc}
0,129 & 0,768 & 0,103 \\
0,012 & 0,742 & 0,245 \\
0,003 & 0,427 & 0,562
\end{array}\right]
$$

Como a matriz NMGI2 possui todas as entradas não nulas, NMGI representa uma cadeia de Markov regular. Pode-se calcular o vetor fixo de probabilidade em longo prazo para o Grau de Inovação das empresas da amostra, utilizando a fórmula (9): 
$\pi \cdot \mathrm{NM}_{\mathrm{GI}}=\pi$

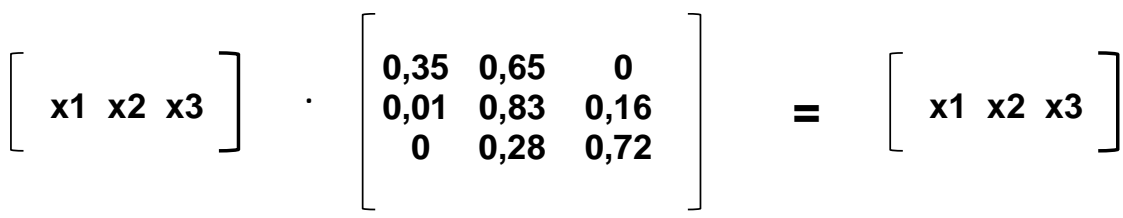

Resolvendo este sistema, são obtidas as seguintes probabilidades:

$$
\mathbf{x} 1=0,01 \quad \times 2=0,63 \quad \times 3=0,36
$$

Portanto, em longo prazo, as empresas tenderão para um Grau de Inovação no nível 2 (63\%) e no nível 3 (36\%). Com esses resultados confirma-se que a amostra representa empresas inovadoras ocasionais. Essas probabilidades refletem uma tendência, caso nenhuma ação do gestor ou evento externo ocorra em longo prazo, as empresas se enquadrarão em uma dessas probabilidades.

Para o Grau Organizacional, seguindo os mesmos passos dos cálculos utilizados para o Grau de Inovação, tem-se em longo prazo as seguintes probabilidades (12):

$$
\begin{gathered}
{\left[\begin{array}{lll}
\text { y1 } & \text { y2 } & \text { y3 }
\end{array}\right] \cdot\left[\begin{array}{ccc}
0,77 & 0,22 & 0,01 \\
0,02 & 0,85 & 0,13 \\
0 & 0,50 & 0,50
\end{array}\right]=\left[\begin{array}{lll}
y 1 & y 2 & y 3
\end{array}\right]} \\
y 1=0,06 \quad y 2=0,74 \quad y 3=0,20
\end{gathered}
$$

Assim, em longo prazo, o Grau Organizacional das empresas da amostra tender-se-á para o nível 2 em 74\%; 20\% para o nível 3 e 6\% no nível 1. Estes resultados apontam que as MPE precisam investir em gestão, repensando o futuro da organização.

Estes resultados corroboram com estudos realizados por Leone (1999) e pelo IBGE (2003) no que tange aos aspectos de caracterização das MPE brasileiras. Ambos revelam traços preponderantes na análise das micro e pequenas empresas: gestão informal, escassez de recursos e baixa qualidade gerencial.

Com relação à análise dos pares GOxGI por empresa, a Tabela 1 representa a matriz de probabilidade de um passo. 
Tabela 1 - Matriz de transição para o par GOxGI

\begin{tabular}{|c|c|c|c|c|c|c|c|c|c|c|c|c|c|c|c|}
\hline & 1,1 & 1,2 & 1,3 & 1,4 & 2,1 & 2,2 & 2,3 & 2,4 & 3,1 & 3,2 & 3,3 & 3,4 & 4,1 & 4,2 & 4,3 \\
\hline 1,1 & 0,35 & 0 & 0 & 0 & 0,62 & 0,03 & 0 & 0 & 0 & 0 & 0 & 0 & 0 & 0 & 0 \\
\hline 1,2 & 0 & 0 & 0 & 0 & 0 & 0 & 0 & 0 & 0 & 0 & 0 & 0 & 0 & 0 & 0 \\
\hline 1,3 & 0 & 0 & 0 & 0 & 0 & 0 & 0 & 0 & 0 & 0 & 0 & 0 & 0 & 0 & 0 \\
\hline 1,4 & 0 & 0 & 0 & 0 & 0 & 0 & 0 & 0 & 0 & 0 & 0 & 0 & 0 & 0 & 0 \\
\hline 2,1 & 0 & 0 & 0 & 0 & 0,60 & 0,27 & 0 & 0 & 0,07 & 0,05 & 0 & 0 & 0 & 0 & 0 \\
\hline 2,2 & 0 & 0,03 & 0 & 0 & 0,03 & 0,67 & 0,03 & 0 & 0,03 & 0,18 & 0,03 & 0 & 0 & 0 & 0 \\
\hline 2,3 & 0 & 0 & 0 & 0 & 0 & 0,67 & 0 & 0 & 0 & 0 & 0,33 & 0 & 0 & 0 & 0 \\
\hline 2,4 & 0 & 0 & 0 & 0 & 0 & 0 & 0 & 0 & 0 & 0 & 0 & 0 & 0 & 0 & 0 \\
\hline 3,1 & 0 & 0 & 0 & 0 & 0 & 0 & 0 & 0 & 0,33 & 0,50 & 0 & 0 & 0 & 0 & 0,17 \\
\hline 3,2 & 0 & 0 & 0 & 0 & 0 & 0 & 0 & 0 & 0 & 0,85 & 0,1 & 0 & 0 & 0 & 0,05 \\
\hline 3,3 & 0 & 0 & 0 & 0 & 0 & 0 & 0 & 0 & 0 & 0 & 1 & 0 & 0 & 0 & 0 \\
\hline 3,4 & 0 & 0 & 0 & 0 & 0 & 0 & 0 & 0 & 0 & 0 & 0 & 0 & 0 & 0 & 0 \\
\hline 4,1 & 0 & 0 & 0 & 0 & 0 & 0 & 0 & 0 & 0 & 0 & 0 & 0 & 0 & 0 & 0 \\
\hline 4,2 & 0 & 0 & 0 & 0 & 0 & 0 & 0 & 0 & 0 & 0 & 0 & 0 & 0 & 0 & 0 \\
\hline 4,3 & 0 & 0 & 0 & 0 & 0 & 0 & 0 & 0 & 0 & 0 & 0 & 0 & 0 & 0 & 0 \\
\hline
\end{tabular}

Fonte: Autor, 2018.

Conforme dados da Tabela 1, analisou-se o perfil das empresas no futuro, utilizando quatro passos a frente do estado atual. Os recortes desses dados estão apresentados na Tabela 2.

Assim, dado que uma empresa esteja no nível de gestão 2 e no nível de inovação 2, as probabilidades futuras mostram que: a empresa tenderá a permanecer no mesmo nível $(2,2)$ em aproximadamente 25\%; migrará para o nível $(2,3)$ com probabilidade de $38 \%$ e para o nível $(3,3)$ com probabilidade de $18 \%$, melhorando, respectivamente, o seu Grau de Gestão de Inovação; cairá para o nível (1,2) com probabilidade de 3\%. Como podemos verificar, a empresa tem maior probabilidade de se manter no nível atual ou melhorar o seu perfil.

Ainda na Tabela 2, verifica-se que uma empresa com nível (2,3), apresenta as seguintes probabilidades: tenderá a permanecer no mesmo nível $(2,3)$ em aproximadamente $52 \%$; migrará para os níveis $(3,3)$ e $(3,4)$ com probabilidades de $32 \%$ e 3\%, respectivamente, melhorando o seu perfil, tanto de gestão, como de inovação. Estes resultados estão mostrados na Tabela 2.

Tabela 2 - Recorte da matriz de transição GOxGI em quatro períodos de tempo

\begin{tabular}{cccccc} 
& 1,2 & 2,2 & 2,3 & 3,3 & 3,4 \\
1,1 & 0,30 & 0,33 & 0,21 & 0,03 & 0,02 \\
\hline 1,2 & 0,15 & 0,30 & 0,32 & 0,08 & 0,02 \\
\hline 2,2 & 0,03 & 0,25 & 0,38 & 0,18 & 0,02 \\
\hline 2,3 & 0 & 0 & 0,52 & 0,32 & 0,03 \\
\hline
\end{tabular}

Fonte: Autor, 2018.

Observa-se, ainda, que a matriz da Tabela 1 possui nove estados absorventes (linhas com todas as entradas nulas). Assim, não se pode utilizar uma forma reduzida da matriz para calcular os estados em longo prazo, assim como fizemos para os cálculos anteriores, uma vez que a mesma não representa uma cadeia de Markov regular. Qualquer potência desta matriz terá entradas nulas. 


\section{Conclusões}

O trabalho abordou as diferentes perspectivas de inovação e formas de mensuração da mesma, começando pelo critério proposto por Schumpeter (1984), chegando às modificações sugeridas por Bachmann (2008), com base nos estudos de Sawhney et al. (2006).

Discorremos, ainda, pelo uso da inovação como estratégia para as empresas obterem vantagem competitiva (Matin \& López, 2007; Tidd et. al. 2005; Alsaaty, 2011).

Motivados pelas discussões relativas à capacidade de inovar das MPE, esta pesquisa buscou descrever e prever os estados futuros das empresas com os dados do grau de desenvolvimento organizacional (GO) e o grau de inovação (GI), a partir do radar de inovação definido por Sawhney et al. (2006) e ampliado por Bachmann (2008).

Utilizamos os resultados do diagnóstico organizacional e de inovação de 60 MPE. A amostra é igualmente distribuída em três grupos de 20 empresas dos setores da indústria de alimentos, móveis e confecções do estado de Pernambuco, selecionadas de modo não probabilístico por adesão dos participantes do projeto Agentes Locais de Inovação (SEBRAE, 2015). Os setores foram escolhidos considerando-se a diferença natural entre eles, sobretudo, no tocante às possibilidades de inovar.

Uma das contribuições deste artigo foi a identificação de uma tendência das empresas da amostra terem perfil de inovadoras ocasionais, de acordo com as probabilidades da cadeia de Markov, calculadas nas matrizes de transição de $\mathrm{n}$ passos de $\mathrm{Gl}$.

Constatou-se, ainda, um incremento nos resultados médios dos graus organizacional e de inovação devido a implantação das melhorias apontadas nos diagnósticos do projeto ALI. Para o Ciclo 0 verificou-se um grau organizacional médio de 1,87 e um grau de inovação médio de 2,47. No Ciclo 1 obtivemos um grau organizacional médio de 1,98 e um grau de inovação médio de 2,69. Para o Ciclo 2 obtivemos um grau organizacional médio de 2,25 e um grau de inovação médio de 2,89. Estes resultados apontam que a ação do ALI nas empresas, melhora a gestão e a inovação, fazendo com que o projeto cumpra a sua missão de tornar as empresas mais competitiva.

Estes resultados corroboram com a teoria de que as MPE buscam formas de inovar que não estão associadas a P\&D, mostrando resultados positivos na implementação de ações que levem as organizações a ocuparem posições superiores aos seus pares (Simantobb \& Lippi, 2003; Bachmann \& Destefanni, 2008; Campos \& Campos, 2013).

O Projeto ALI (SEBRAE, 2015) conseguiu incrementar o grau organizacional e o grau de inovação da amostra pesquisada, porém não constatou-se que essas empresas se tornaram inovadoras sistêmicas após a implementação das melhorias apontadas nos diagnósticos. O grande desafio das organizações é implantar a cultura da inovação nas empresas, de tal forma que as MPE consigam permanecer inovadoras, mesmo após o término dos programas de fomento. É de fundamental importância avaliar se os modelos utilizados por esses programas conseguem gerar um diferencial competitivo nas MPE.

Uma proposta de trabalho futuro é a proposição de uma nova taxonomia para as MPE, baseada no grau de inovação. Hoje as empresas são classificadas pela quantidade de funcionários ou faturamento. Essa nova taxonomia permitiria a utilização de planos de ações e políticas públicas direcionados para tornar as MPE mais 
inovadoras em um menor espaço de tempo, aumentando a sua vantagem competitiva e sobrevivência no mercado.

Espera-se que os resultados desta pesquisa contribuam para as ações dos gestores das empresas, além de promover uma reflexão para a implantação de políticas públicas direcionadas às empresas de pequeno porte no tocante à inovação.

Referências

Akis, E. (2015). Innovation and Competitive Power. Procedia-Social and Behavioral Sciences, 195, 1311-1320.

Alsaaty, F. M. (2011). A model for building innovation capabilities in small entrepreneurial firms. Academy of Entrepreneurship Journal, 17(1).

Andreasi, T.; \&SBragia, R. (2002) Fatores determinantes do grau de novatividade das empresas:um estudo utilizando a técnica de análise discriminante. Working Papers n. 001/004, FEA-USP.

Andreasi, T. 2003. Innovation in small and médium-sized enterprises. International Journal of Entrepreneurship and Innovation Management, v. 3, n. 1/2.

Bachmann, D. 2008. Agentes locais de inovação. Uma medida do progresso nas MPE do Paraná. Paraná: Sebrae.

Campos, L. B. P.; \& Campos, R. J. (2013). Análise multi-casos da gestão da inovação em empresas de pequeno porte. Pretexto, Belo Horizonte, v. 14, n. 1, p. 36-51.

Estrada, M. A. L. 2015. Teoremas limiares para o modelo SIR estocástico de epidemia.39 f. Dissertação (mestrado) - Universidade Estadual de Campinas, Instituto de Matemática Estatística e Computação Científica, Campinas, SP. Disponível em: <http://www.bibliotecadigital.unicamp.br/document/?code=000946406>. Acesso em: 2 maio. 2017

Fidalga, N. H. D.. 2016. Aplicações das cadeias de markov à modelação matemática de epidemias. Dissertação (mestrado). Instituto Politécnico de Bragança - Escola Superior de Tecnologia e Gestão. Portugal.

Fórum Permanente das Microempresas e Empresas de Pequeno Porte. 2007. Desenvolvimento tecnológico e inovação nas micro e pequenas empresas: fatores de influência. São Paulo.

Fundação Nacional da Qualidade. 2016. Modelo de Excelência da Gestão (MEG). Recuperado em 02 de maio de 2017. https://www.fnq.org.br/guia_referencia_MEG_21_abril_16.pdf

Gamal, D., Salah ,T., \& Elrayyes, N. (2011). "How to measure organization Innovativeness." Technology Innovation and Entrepreneurship Center.

IBGE. As Micro e Pequenas Empresas Comerciais e de Serviços no Brasil. https://ww2.ibge.gov.br/home/estatistica/economia/microempresa/default.shtm. Acessado em 25/01/2018.

Leone, N.M. (1999). As especificidades das pequenas e médias empresas. São Paulo: Revista de Administração, v.34, n.2, p.91-94.

Leone, R. J. G.; \& Leone, N. M. C. P. G. (2012). Pequenas e médias empresas: contribuições para discussão sobre por que e como medir o seu tamanho. Revista do Mestrado em Administração da Universidade Potiguar RaUnP - Ano 4, n.1.

Manual de Oslo. 2004. Proposta de diretrizes para coleta e interpretação de dados sobre inovação tecnológica. Organização para Cooperação Econômica e desenvolvimento: Versão FINEP.

Marcos, W.P. 2014. "Cadeia de Markov aplicada ao manejo de pragas em lavoura cafeeira." Universidade Federal de Uberlândia - M.G..

Martín, L. A., \& López, J. M. (2007). La Dirección Estratégica de la Empresa: teoria y aplicaciones. Pamplona: Editorial Aranzadi.

Nuñez, J. L.T. (2004). "Modelo para o gerenciamento de ativos de transmissão de energia elétrica." Universidade Federal de Santa Catarina, Centro Tecnológico. Programa de Pós-Graduação em Engenharia Elétrica.

Oliveira, J.; Terence, A. C. F.; \& Escrivão Filho, E. (2010). Planejamento Estratégico e Operacional na Pequena Empresa: Impactos da formalização no desempenho e diferenças setoriais. Revista Gestão Organizacional RGO. v 3, n. 1.

Oliveira, P. G. O. (2016). Estudo de Confiabilidade de Sistemas de Controle de Dispositivos de Segurança de Subsuperfície em Poços de Petróleo. Dissertação de Mestrado. PUC Rio.

Pesquisa Industrial Inovação Tecnológica - PINTEC. (2005). Instituto Brasileiro de Geografia e Estatística. Instituto Brasileiro de Geografia e Estatística - IBGE, Rio de Janeiro, 2006. Disponível em: 
<http://www.pintec.ibge.gov.br/downloads/PUBLICACAO/Publicacao\%20PINTEC\%202005.pdf>. Acessado em: 25/02/2018.

Reichert, F., Camboim, G., \& Zawislak, P. (2015). Capacidades e Trajetórias de Inovação de Empresas Brasileiras. Ram-Revista de Administração Mackenzie, 16(5),( pp.161-194).

Ross, S. M. (2003). "Introduction to Probability Models", McGraw-Hill, 801 p.

Sawhney, M., Wolcott, R. C., \& Arroniz, I. 2006. The 12 different ways for companies to innovate (v. 47, n. 3). MIT Sloan Management Review (pp. 75-81).

Schumpeter, J.A. (1984). The theory of economic development: an inquiry into profits, capital, credit, interest, and the business cycle. New York: Oxford University Press.

Serviço Brasileiro de Apoio às Micro e Pequenas Empresas (SEBRAE). (2014). Participação das Micro e Pequenas Empresas na Economia Brasileira. Recuperado em: 30 janeiro de 2018. < https://m.sebrae.com.br/Sebrae/Portal\%20Sebrae/Estudos\%20e\%20Pesquisas/Participacao\%20das\%20micro $\% 20$ e\%20pequenas\%20empresas.pdf>.

Serviço Brasileiro de Apoio às Micro e Pequenas Empresas (SEBRAE). (2015). Projeto agente local de inovação (ALI) em Pernambuco. Recuperado em: 01 maio de 2017. <

https://www.sebrae.com.br/sites/PortalSebrae/ufs/pe/sebraeaz/agentes-locais-deinovacao,ad4f1a5f5387e410VgnVCM1000003b74010aRCRD>.

Silos, Pedro. (2006). Assessing Markov chain approximations: A minimal econometric approach. Journal of Economic Dynamics and Control, v. 30, n. 6, )pp. 1063-1079).

Sullivan, M.; \& Mizrahi, A. (2006) Matemática finita: Uma Abordagem Aplicada. 9 ed. Rio de Janeiro: LTC. Simantob, M.; \& Lippi, R. (2003). Guia Valor Econômico de Inovação nas Empresas, Editora Globo, São Paulo. Taha, H. (2008). A. Pesquisa Operacional. 8 ed. São Paulo: Pearson Prentice Hall. 361 p.

Tidd, J., Bessant, J., \& Pavitt, K. (2008). Gestão da Inovação. (3.ed.). Porto Alegre: Bookman.

Titu, A., Raulea, A., \& Titu, S. (2015). Innovation-a Challenge for the 21st. Century Managers. Economics and Finance, 27, (pp. 126-135).

Vargas, H. C., Estrada, S., \& Gómez, E. L. (2016) The effects of ICTs as innovation facilitators for a greater business performance. Evidence from Mexico. Computer Science, 91, (pp. 47-56).

Zehir, C., Köle, M., \& Yıldız, H. (2015). The Mediating Role of Innovation Capability on Market Orientation and Export Performance: an Implementation on SMEs in Turkey. Procedia-Social and Behavioral Sciences, 207, (pp.700-708).

Recebido em: 31 jan. 2018 / Aprovado em: 20 ago. 2018

\section{Para referenciar este texto}

Silva, A. M. da., \& Cavalcanti, A. M. (2020). Predição da gestão organizacional e do perfil inovador das empresas de pequeno porte: análise à luz de Cadeias de Markov. Exacta, 18(2), 244-258. https://doi.org/10.5585/ExactaEP.v18n2.8313. 